\title{
Microvascular Characteristics of Auditory Cortex: A Corrosion Cast Study
}

\author{
A.T.D. Licup-Bravo, ${ }^{*}$ R.J. Mount, ${ }^{*}$ B. Minnich, ${ }^{* *}$ A. Lametschwandtner, ${ }^{* *}$ and R.V. Harrison* \\ * Auditory Science Laboratory, Brain and Behaviour Division and Department of Otolaryngology, \\ Hospital for Sick Children, 555 University Ave., Toronto, Canada M5G 1X8 \\ ** Department of Organismic Biology, Vascular and Muscle Research Group, University of \\ Salzburg, Hellbrunnerstrasse 34, A-5020, Salzburg, Austria
}

Sound energy reaching the ears is coded and transmitted via the auditory neural pathway to the temporal lobe of cortex. The primary area of auditory cortex is characterized by neurons which respond with shorter latency and generally higher levels of activity than those in surrounding secondary or association areas. The metabolic need of these neurons is provided for, in part, by the local blood supply at the capillary bed level. Blood supply to auditory cortex is via a branch of the medial cerebral artery. Descriptions of microvessel architecture in the brain have mostly been based on two-dimensional histological studies. Corrosion casting techniques provide accurate 3-D models of the vasculature which can be viewed with a scanning electron microscope (SEM) to provide details at the capillary level $[1,2]$.

The present study investigates whether the different metabolic demands of neurons in primary vs. non primary regions require different blood supply systems. Our study objectives were to characterize the microvascular anatomy in the auditory cortex in the chinchilla, and to quantify vascular properties within the active primary cortex (AI) and the less active peripheral regions (nonAI). The parameters of vascularity which were used to compare AI and nonAI were: capillary diameter, number of capillary segments, segment lengths, number of branching points and the branching angles and intervascular distances.

Twelve adult chinchillas underwent electrophysiologic recordings of exposed auditory cortex to identify a functional map of AI (Fig. 1A). 3-D casts of auditory cortex vasculature were prepared by corrosion cast method, and SEM images obtained. Surface vasculature seen in the electrophysiologic maps was aligned with that of the corrosion cast to identify AI region (Fig. 1B). Stereopair scanning electron micrographs, which provide depth of field and the ability to trace vessels along 3 axes, were taken from both the AI and nonAI regions (Fig. 1C). Vascular parameters were determined by using an automated quantitative analysis system [3].

Mean capillary diameters were higher in AI $(\mathrm{p}=0.0139)$. Parameters of microvessel density (number of capillary segments and branching points) were significantly higher in AI ( $p=0.0017$ and 0.0002 respectively). Intervascular distance is less in AI compared to nonAI regions $(p<0.0001)$. Segment length and branching angles were not different between the groups (Table 1).

Corrosion casting combined with electrophysiologic mapping allows for correlated analysis of the relationship between local neural function in cortex and blood supply. In primary auditory cortex, a highly active sensory region, capillaries are more densely packed, have a greater diameter and exhibit more branching compared to those in adjacent, but less active, non primary areas. 
References

[1] J Panesar, H Hamrahi, N Harel, RJ Mount and RV Harrison, Acta Otolaryngol. 121(2001) 839.

[2] RV Harrison, N Harel, J Panesar and RJ Mount, Cereb Cortex. 12 (2002) 225.

[3] B Minnich, H Leeb, EWN Bernroider and A Lametschwandtner, J Microsc. 195 (1999) 23.

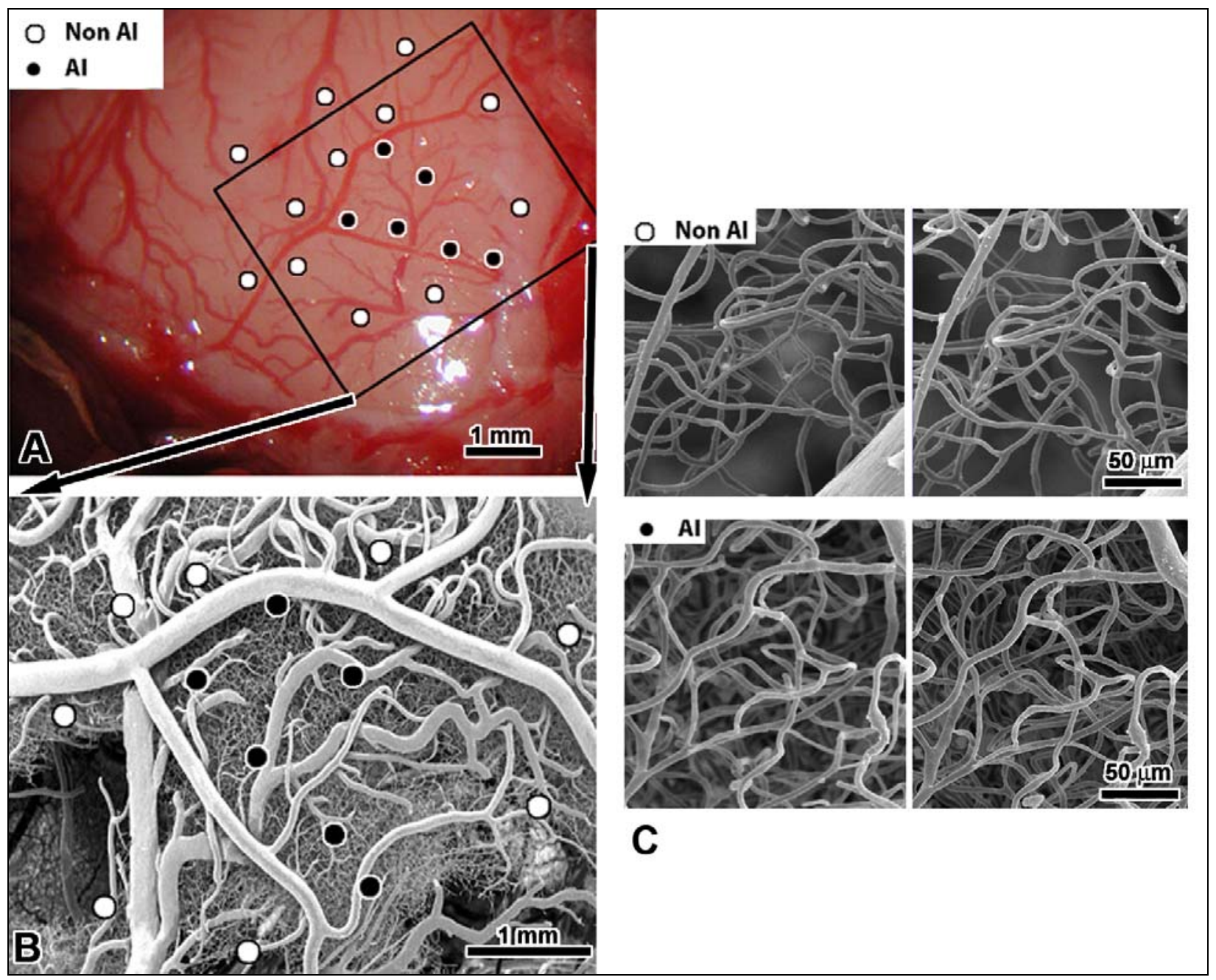

Fig. 1. A) Electrophysiologic mapping via craniotomy; B) Superimposition of map onto photomicrograph of cast; C) Stereopair images of capillaries in AI and nonAI regions.

TABLE 1. Vascular parameters of AI and nonAI regions (* significant difference).

\begin{tabular}{lrrr}
\hline Vascular Parameters & AI & nonAI & T test \\
\hline Capillary Diameters $(\mu \mathrm{m})$ & 3.28 & 3.20 & $\mathbf{0 . 0 1 3 9 *}$ \\
Segments / 0.1 sq.mm & 206.33 & 184.05 & $\mathbf{0 . 0 0 1 7 *}$ \\
Branching Points / 0.1 sq.mm & 94.90 & 83.08 & $\mathbf{0 . 0 0 0 2 *}$ \\
Segment length $(\mu \mathrm{m})$ & 63.11 & 64.26 & 0.3481 \\
Intervascular distance $(\mu \mathrm{m})$ & 38.45 & 45.58 & $<\mathbf{0 . 0 0 0 1 *}$ \\
Branching angle $\left({ }^{\circ}\right)$ & 70.43 & 70.94 & 0.6438 \\
\hline
\end{tabular}

